\title{
Implementation and investigation of a force control for a forming demonstrator with coupled servo drives
}

\author{
André Sewohl ${ }^{1, *}$, Armin Schleinitz ${ }^{1}$, Holger Schlegel ${ }^{1}$ and Matthias Putz ${ }^{2}$ \\ ${ }^{1}$ Chemnitz University of Technology, Department of Control and Feedback Control Technology, 09126 Chemnitz, Germany \\ ${ }^{2}$ Fraunhofer Institute for Machine Tools and Forming Technology, Production Department, 09126 Chemnitz, Germany
}

\begin{abstract}
In the area of production engineering, there are ongoing efforts to improve manufacturing strategies and processes in terms of stability, quality and efficiency. One possibility for ensuring stable process conditions and reducing rejected parts is closed loop control of quality determining parameters. For many applications, the machining forces often represent the limiting factor for the design of the processes and the choice of parameters. As a controlled value, they are predestined for ensuring process stability and safety. For the tasks and problems of forming technology, control of process forces is not yet mature and offers significant potential for improvement. The drafting of sophisticated control concepts, which enable a mutual use or a combination of force and position control, is the focus of future developments. The design and implementation of a force control for a forming demonstrator with coupled servo drives is described in this publication. The combination of force and position control is realized by direct switching. In addition, further combination options and structures are explained. The focus here is first on the parameterization of the force control and the assessment of the control performance using appropriate comparison criteria in the time domain.
\end{abstract}

\section{Introduction}

In modern production systems, there is a trend to replace mechanical motion solutions with electrical ones. There are many strategies for controlling machine-specific quantities, such as the position or speed of electromechanical axes. The concept of cascade structure, also called servo control, has become established in this field [1]. The use of controlled electromechanical drive systems can meet the increasing demands on the machines in terms of dynamic behavior, as well as higher productivity and accuracy.

Nevertheless, in the area of production engineering, there are ongoing efforts to improve manufacturing strategies and processes in terms of stability, quality and efficiency. One possibility for ensuring stable process conditions and reducing rejected parts is closed loop control of quality determining parameters [2]. The development of suitable control concepts at the process level, in which significant process variables are taken into account as controlled values, offers considerable room for improvement at this point. Process control in terms of manufacturing technology corresponds to influence process variables for controlling the process $[3,4,5]$. This requires the integration of sensors for the detection of the current process status.

Besides the determination of state variables, the use of sensors also makes it possible to set up additional control loops which react to changing boundary conditions in the process and allow specific manipulation. This is the basis for the development of intelligent production machines, which react to unknown disturbances and automatically adapt to process fluctuations. As a result, even complex manufacturing processes can be safely operated at their technological limits and productivity as well as process reliability can be further increased.

At the higher process level, suitable variables must be selected to influence the process. There are many process variables which have an influence to the quality of a part. However, usually it is very difficult to control these values. The metrological acquisition of corresponding parameters constitutes a further challenge. But it is necessary in order to obtain precise knowledge about the current process status. For a large part of the processes in the field of production technology, the machining forces are of particular relevance. As a controlled variable, they are predestined for ensuring process stability and safety. Machining forces are often the limiting factor for the design of the processes and the choice of parameters. Excessive loads can cause damage and defects to the workpiece, tool or machine. In the worst case, they even lead to their destruction. In addition, process forces provide important information about the process state and allow conclusions about deviations in the production process, the machine, the tool, the workpiece or material.

Particularly in processes from the field of machining technology [3] and forming technology [2], the targeted influencing of process forces is of outstanding

Corresponding author: andre.sewohl@mb.tu-chemnitz.de 
importance. In the area of metal-cutting manufacturing processes, a large number of concepts and algorithms for controlling the process forces were investigated and developed on both the research and industry side $[6,7]$. Also in the area of forming technology extensive investigations took place. Nevertheless, there is still a clear need for action in the future, as shown in [2].

In the next chapter, the concept of hybrid forceposition control will be presented as one possible approach. This is followed by a description of the selected test rig for forming technologies, with regard to its mechanical structure and the existing control architecture. Subsequently, a force control is designed for this demonstrator as a first step on the way to the hybrid force position control. Finally, various adjustment rules are experimentally investigated and compared on the basis of corresponding quality criteria.

\section{Control Concept}

While the control of process forces has considerable potential for improvement, in many applications and processes the position control is at the same time essential for compliance with defined position specifications. Thus, the drafting of sophisticated control concepts, which enable joint use or the combination of force and position control, is the focus of future developments. For the tasks and problems of production engineering, the control of process forces is not yet mature and offers significant room for improvement. The concept of a hybrid force position control represents a promising approach at this point. The basic structure and functionality is illustrated in Figure 1.

The core component of the concept is the simultaneous usage and operation of a force and position controller. Various control algorithms and controller structures can be used here. It makes sense to implement the position control for electromechanical drive systems in the form of the established and extensively studied cascade structure. A variety of different embodiments comes in question for the force controller. These range from simple PID-controllers to complex methods from the field of so-called higher control technology. For example, adaptive or predictive controllers, as well as artificial neural networks or fuzzy controllers in the domain of cognition. Also a linkage and combination of the different methods is conceivable. The individual areas and variants were presented in more detail in [8].

For use in production machines, suitable concepts that allow simultaneous control of force and position still need to be developed. The main challenges are the concurrent use of the controllers, the selection of design principles and structures as well as the fusion of the two manipulated variables. One possible approach is to introduce a weighting function that prioritizes the controllers and their manipulated variables. To clarify these questions, extensive investigations are necessary. Against this background, simulation models are helpful, which can be successively extended to examine the complex relationships step by step. On the basis of the models, possibilities for the combination of force and position control should be considered. Starting point for this is a forming demonstrator with coupled drives. A servo control in cascade structure was initially designed for the test rig so that it can be operated in a positioncontrolled manner. The next chapter will give a more detailed description.

The demonstrator will be used for the field trial and practical implementation of the concepts, as well as the examination of the feasibility and limitations. As a first step, the enhancement of the control structure with a force controller takes place. At the test rig, the design is

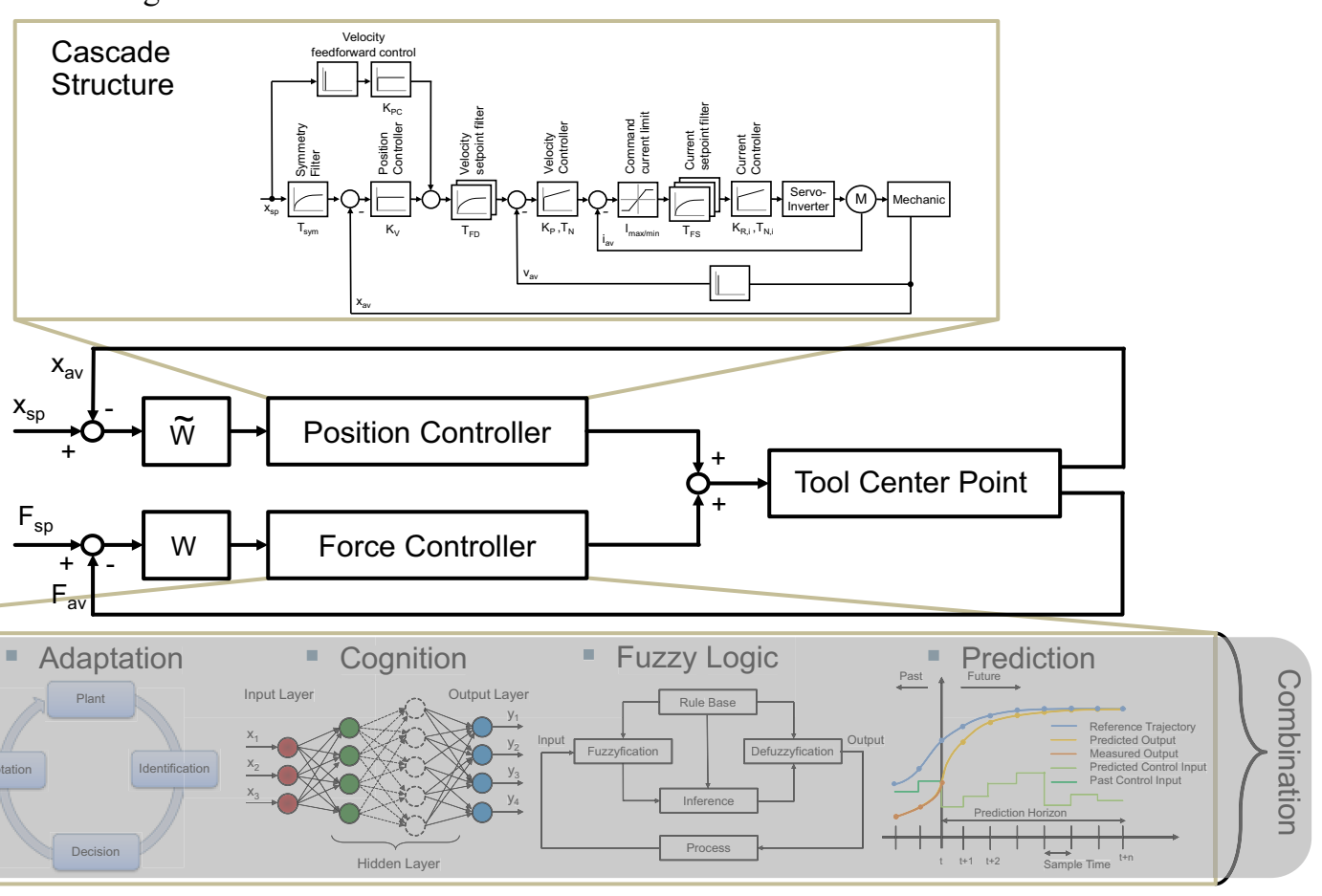

Fig. 1. Schematic concept for hybrid force-position control. 
initially carried out with a PID-structure for the force controller. There are various options for integrating the controller in the existing architecture, which are explained in more detail in Chapter 4. In addition, the combination with the position control is realized by a switchover for experimental proving.

\section{Servo forming unit}

\subsection{Mechanical components and structure}

The core component of the investigations is a self-driven press tool, which offers a very high degree of flexibility and process stability. The basis construction of the demonstrator is similar to a servo screw press with two drives. A general description of the demonstrator with its set-up of basis tool, single modules, actuators and control technology is given in [9]. The schematic structure of the mechatronic system is shown in Figure 2. The mechanical components are illustrated as CADmodel and electrical components as well as the control structure are described in more detail in the following sections.

The basic tool (grey) consists of two plates, which are connected to the direct driven actuators (blue) on opposite sides. The top plate is coupled via tilting joints with the two drives. A piezoelectric measuring ring (green) is integrated in each drive train, in order to detect the force. Parallel to the actuator-basis tool-connection two linear measuring systems (also green) are utilized to monitor the relative movement of the upper and lower plate of the basis tool. This value can be used in the control concept in order to avoid unwanted tilt. Additionally a column guide with four elements (pink) is mounted in the workspace. This is a sliding guide with a diameter of $25 \mathrm{~mm}$ and with a backlash between $18 \mu \mathrm{m}$ to $25 \mu \mathrm{m}$. Both servo-electric motors are driven by individual drive units and offer a maximum in power and momentum at every position, which is required to avoid tilt. Since tilt is not avoided by the guiding elements of their own, maximum forming force depends on the load eccentricity. The influence of an eccentric load case on the basis module can be evaluated with the demonstrator. The single load modules (brown) can be placed eccentrically as well as centrally in the basis module. Thus an unbalanced load can cause tilt in the guidance which needs to be compensated by the feedback control system. Furthermore it is possible to remove the guiding elements to allow a larger tilt. The single load modules are different in their construction and accordingly different in their force-displacement-behavior. The force-stroke curve from these modules is so far adaptable that several real processes can be replaced perfectly. Major advantages are an exact reproducibility, no need in further material and the force-stroke curve can be adjusted individually for many kinds of tools. For example, the spring modules are comparable to real processes like deep drawing or bending and replace real deep drawing or bending tools.

\subsection{Electrical components and structure}

The core components of the demonstrator are the two linear actuators of the type GSX 40-0601-MFA-IG4258-SR-RB manufactured by Exlar. In order to generate the linear movement, planetary roller screws are integrated directly in the drive. They possess a larger contact surface, as well as a longer lifetime and higher load than a ball screw system. The schematic construction is shown in Figure 3.

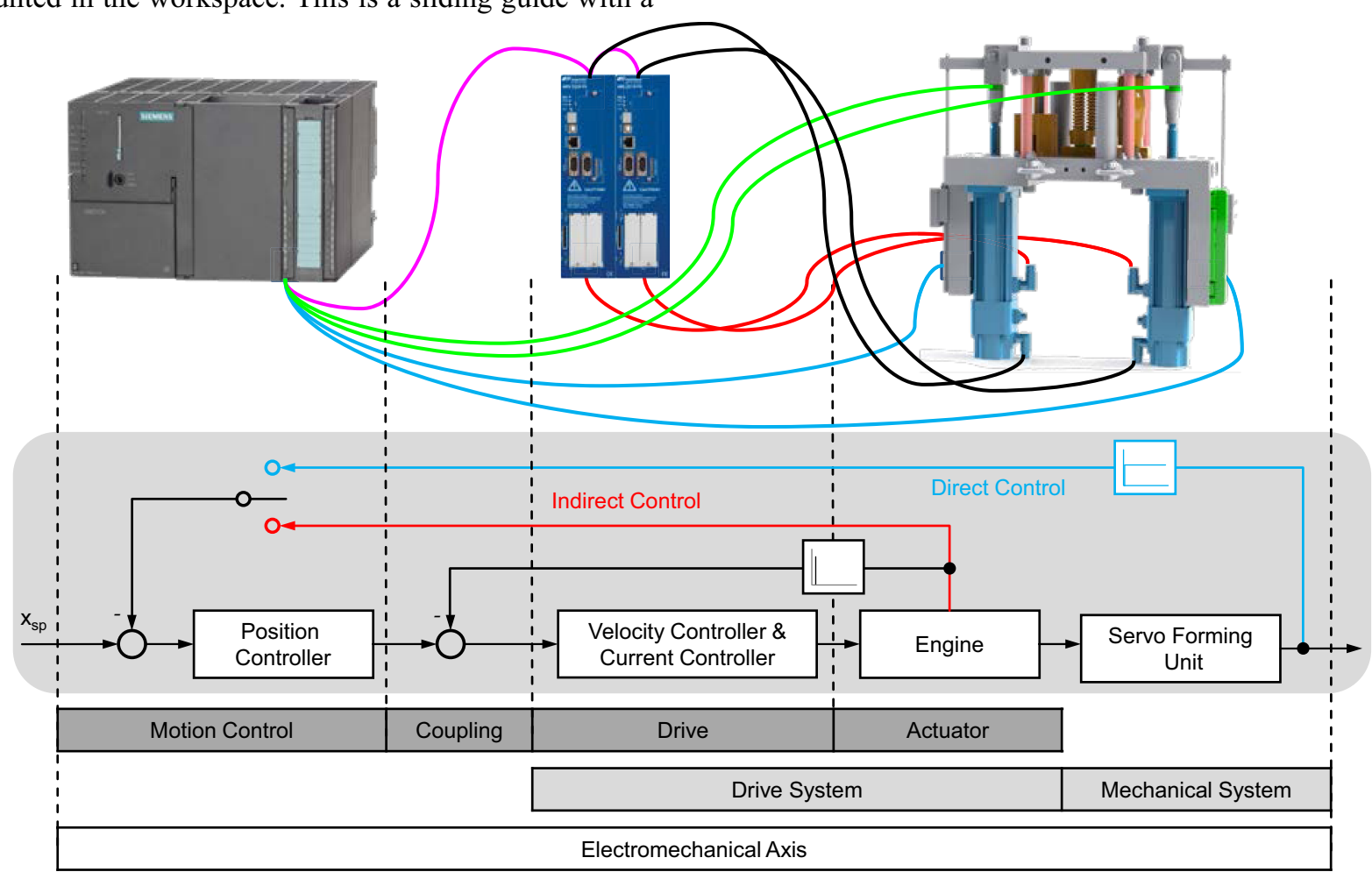

Fig. 2. Mechatronic system of the demonstrator. 


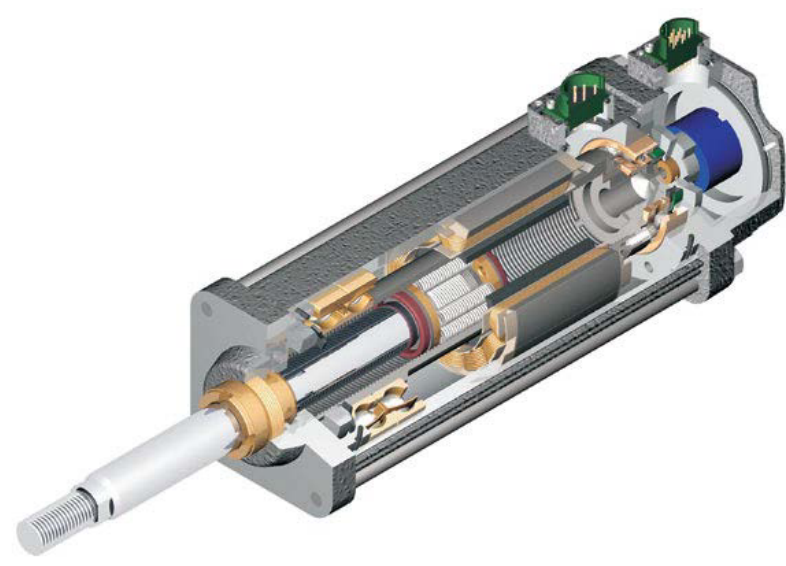

Fig. 3. Linear actuator with planetary roller screw [10].

The actuators are each connected to an ISR 2310-FS servo-controller from Metronix. These drive controllers are characterized by the smallest possible cycle times in the two-digit $\mu$ s range up to the speed and position controller level. This allows a very fast system response, which is especially required for force control. Furthermore, that ensures a parallel position in the tool plane. In the field of control technology, this can only be realized with low dead times by fast clocking, in order to ensure a robust velocity controller with dynamic setting. A Motion Control Simotion C240 from Siemens is superimposed on the inverters. The movement specification and position control takes place here. In addition, the control of the mechanically coupled drives is implemented via an appropriate interface as axis arrangement with a master-slave structure. In this way, synchronization functions can be realized and the slave drive receives its setpoints from the master axis and follows them via its internal control. Although a direct control of parallelism is not possible in these functions, this configuration is a common implementation for an operation with synchronous motion. A superimposed parallelism control includes delaying communication times. That means their advantages are compensated in this setup by small delay times.

The use of a superordinated motion control ensures a maximum in flexibility. The Simotion C240 enables the integration of additional force sensors and position measuring systems. An adaptation and extension of the control structures can also be applied. Because of extensive I/O-modules, the sub-functions interpolation, position control and speed control can be divided as required between the superordinated motion control and the drive controllers from Metronix. In addition, the appropriate Simotion Scout software from Siemens is available for the operation, configuration and parameterization of test series. The program also offers possibilities for recording and evaluating the measuring signals.

The incremental position measuring systems LS177 from Heidenhain are connected to the Simotion C240 over digital I/O modules. They possess a resolution of $1 \mu \mathrm{m}$. This enables the implementation of a direct position control. Furthermore, piezoelectric measuring rings 9051A from Kistler are preloaded in the power flow of the push rods. Their measurement signals are evaluated via a permanently installed industrial 2channel charge amplifier. This one is also connected to the C240, so that the measuring signals can be used for a force control.

\subsection{Commissioning of the test rig}

In a first step, a position control in cascade structure was first implemented for the commissioning of the demonstrator and the execution of practical experiments with the load units. As a result, real machining processes can be simulated. At this point, the variant was selected, that interpolation and position control takes place on the motion control. The velocity and current control is implemented in each of the drives. This has the advantage of a flexible parameterization and data acquisition. The commissioning of a cascade control always takes place starting with the inner control loop outwards. In this context, the first step was to configure the parameters of the feed axes based on the motor data. This was followed by the parameterization of the current control loop. Based on this, the speed control loop could be designed. The automatic controller tuning and optimization system of the commissioning tool from the drive controllers was used for this purpose. The individual drives were not mechanically linked at this point. The determined parameters of the control loops are summarized in Table 1.

Table 1. Settings of the closed-loop control system.

\begin{tabular}{|c|c|c|c|c|}
\hline \multirow{2}{*}{} & Amplification constant & $\mathrm{K}_{\mathrm{P}}$ & 25 & {$[\mathrm{Nms} / \mathrm{rad}]$} \\
\cline { 2 - 5 } & integration constant & $\mathrm{T}_{\mathrm{n}}$ & 2.5 & {$[\mathrm{~ms}]$} \\
\cline { 2 - 5 } & $\begin{array}{r}\text { actual value filter time } \\
\text { constant }\end{array}$ & $\mathrm{T}_{\mathrm{f}, \mathrm{av}}$ & 0.21 & {$[\mathrm{~ms}]$} \\
\cline { 2 - 5 } & $\begin{array}{c}\text { Synchronous speed } \\
\text { filter time constant }\end{array}$ & $\mathrm{T}_{\mathrm{f}, \mathrm{syn}}$ & 0.6 & {$[\mathrm{~ms}]$} \\
\hline \multirow{2}{*}{\begin{tabular}{c} 
ing \\
\cline { 2 - 5 }
\end{tabular}} & Amplification constant & $\mathrm{K}_{\mathrm{i}}$ & 7.1 & {$[\mathrm{~A} / \mathrm{Nm}]$} \\
\cline { 2 - 5 } & integration constant & $\mathrm{T}_{\mathrm{ni}}$ & 1.46 & {$[\mathrm{~ms}]$} \\
\hline
\end{tabular}

The position control was designed for aperiodic transmission behavior with a damping of $\mathrm{D}=1$ in order to avoid overshooting. For this purpose, [11] proposes the calculation of the gain factor $\mathrm{K}_{\mathrm{v}}$ according to the following formula:

$$
K_{\mathrm{v}}=1 /\left(4 * T_{\text {sum }}\right)
$$

Here, $\mathrm{T}_{\text {sum }}$ corresponds to the total time constant, into which the time constants and dead times of the subordinated current and speed control loops are incorporated. It amounts to:

$$
T_{\text {sum }}=T_{\text {dead }}+\left(T_{\mathrm{r}}-T_{\text {dead }}\right) / 3+T_{\text {sub }}
$$


The corresponding time constants were determined experimentally and are given in Table 2 . In addition, the position control has been supplemented with a velocity feed forward control in order to improve the dynamic behavior and to reduce the contouring error. For this, the weighting factor $\mathrm{K}_{\mathrm{pc}}$ and the symmetry filter time constant $\mathrm{T}_{\text {sym }}$ must be specified. In this regard, no sizing rule has yet been established. There are only suggestions within certain limits, so that offers an empirical dimensioning. The parameters selected for this specific application are listed together with the calculated characteristic values in Table 2.

The resulting controller structure is shown schematically in Figure 2. In this configuration, forming processes respectively their force-displacement behaviour can be simulated with the demonstrator. An extension of the controller structure is necessary for the force-controlled operation. The approach followed is described in the next section.

Table 2. Settings of the position closed-loop control system.

\begin{tabular}{|c|c|c|c|}
\hline Amplification constant & $\mathrm{K}_{\mathrm{v}}$ & 45 & {$[1 / \mathrm{s}]$} \\
\hline Dead time & $\mathrm{T}_{\text {dead }}$ & 2 & {$[\mathrm{~ms}]$} \\
\hline Substituted time constant & $\mathrm{T}_{\text {sub }}$ & 2.7 & {$[\mathrm{~ms}]$} \\
\hline Control rise time & $\mathrm{T}_{\mathrm{r}}$ & 4.4 & {$[\mathrm{~ms}]$} \\
\hline $\begin{array}{c}\text { Weighting factor } \\
\text { Synchronous filter time } \\
\text { constant }\end{array}$ & $\mathrm{K}_{\mathrm{pc}}$ & 60 & {$[\%]$} \\
\hline $\begin{array}{c}\text { Total time constant } \\
\text { syn }\end{array}$ & 3.3 & {$[\mathrm{~ms}]$} \\
\hline Tum & 5.5 & {$[\mathrm{~ms}]$} \\
\hline
\end{tabular}

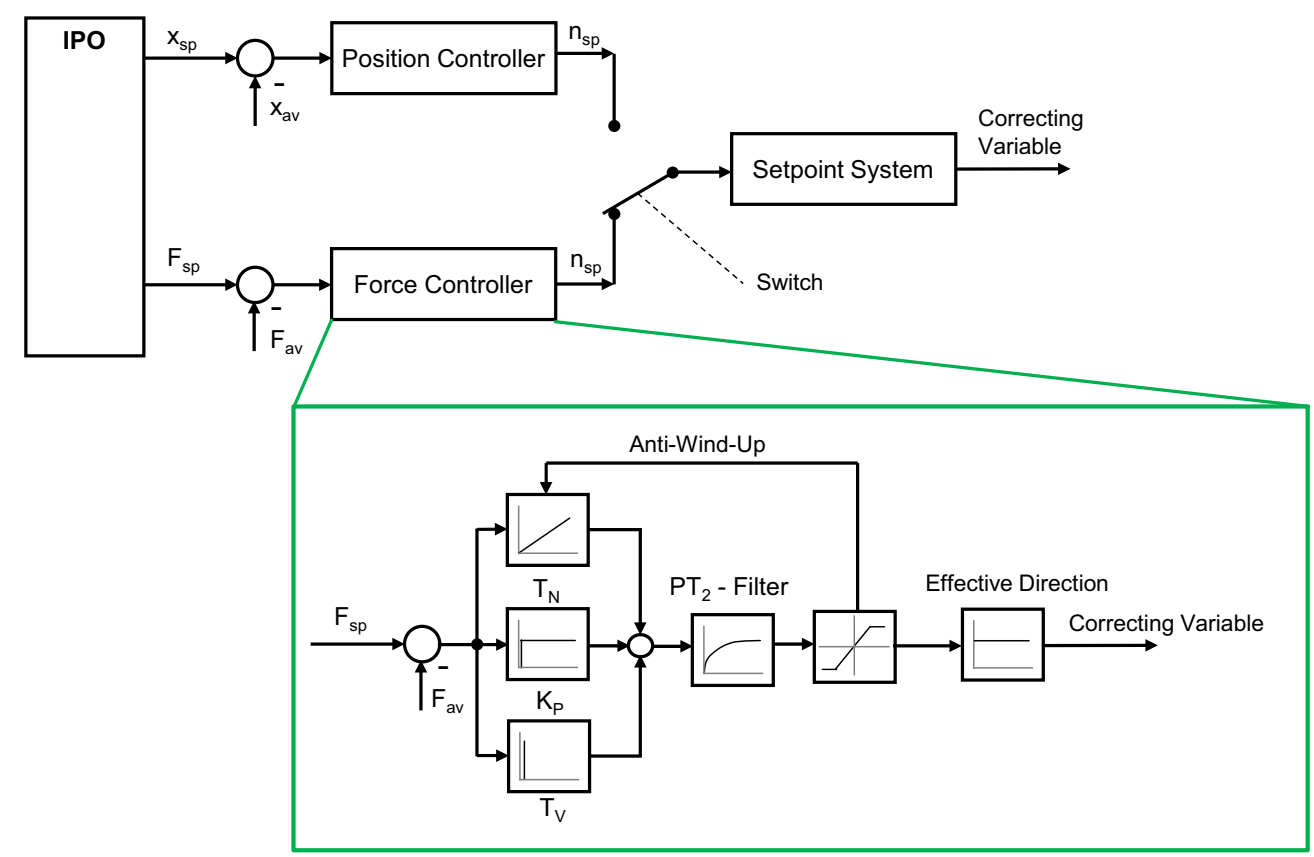

Fig. 4. Changeover and structure of force controller. 
variable. As a result, the velocity or speed continues to be transferred as the setpoint for the subordinate control loops in the inverter. The basic structure is shown in Figure 4.

For switching from position-controlled movement to the force-controlled state and vice versa, corresponding criteria must be defined. These can be defined in specific activation commands. The activation can be done directly or automatically thereby. Direct activation is only possible when the machine is at a standstill with activated position controller. Furthermore, the force control can be automatically activated with a corresponding condition. For automatic activation, a separate command is available. A check of the conditions and the switching takes place in the servo clock. Possible conditions are force, position, time and input values. These parameters can be specified and linked in several stages with the command. By cancelation of the command before the conditions are met, the conditions can also be switched in operation. Parameters are stored at the moment of switching. These values are available through the system variables. The force setpoint or profile specification is then also made via corresponding commands in the C240. There, the force control can be realized via an adjustable PIDcontroller with filter and anti-wind-up. The corresponding structure is illustrated in Figure 4.

The I-component of the controller is initialized in such a way that the new manipulated variable from the force controller corresponds to the estimated manipulated variable from the position controller, in order to obtain a constant manipulated variable when switching from position control to force control. The estimated manipulated variable is the last manipulated variable of the position controller plus the filtered actual acceleration multiplied by the cycle time. In order to obtain a continuous curve of the manipulated variable when switching from force control to position control, the setpoint generation and the position controller are reset. The currently actual value, measured by the encoder, is the new value for the position setpoint, modified by the estimated contouring error. The estimated contouring error is calculated from the actual manipulated variable, multiplied by the substituted time constant of the position control loop. Then, the position controller outputs the same manipulated variable in the first cycle after switching as the force controller before switching over.

\subsection{System identification}

For the design and parameterization of the controller, it is necessary to perform a system identification. In force control, the process which causes the load is part of the controlled system and must be included in the design. Thus, the controlled system is composed of the current and speed control loop, the mechanical components and the process. The spring pack was selected and used in order to simulate a load situation respectively a forming process. The introduction of the load is done centric, so that the forces are evenly distributed on both drives.

The identification and excitation of the controlled system takes place by means of a jump function. Setpoint value is the velocity, that is the manipulated variable of the force controller. For practical execution, the position controller was deactivated and the drives were decoupled. The velocity jump was switched to both drives simultaneously by a setpoint input. So that static friction effects are not included in the identification, both drives were initially moved at a speed of $1 \mathrm{~mm} / \mathrm{s}$. Then the jump of $10 \mathrm{~mm} / \mathrm{s}$ took place. In accordance with this procedure, the step response of drive 1 is shown in Figure 5.

It can be clearly seen that this is a controlled system with integrating behavior and one delaying time constant. Since the movement takes place downwards and there are pressure forces, these are associated with a negative sign. Accordingly to Figure 5, characteristic parameters of the controlled system can be determined. The gain factor $K_{S I}$ is here $170 \mathrm{~N} / \mathrm{mm}$ and the delaying time constant $T_{u}$ is $10 \mathrm{~ms}$. For drive 2 the same values could be determined. Thus, the theoretically known spring constant of $333 \mathrm{~N} / \mathrm{mm}$, which is split between the two drives during a load case, could also be validated. Based on these characteristics, the controller parameters can be calculated according to various adjustment instructions, which are described in the next section.

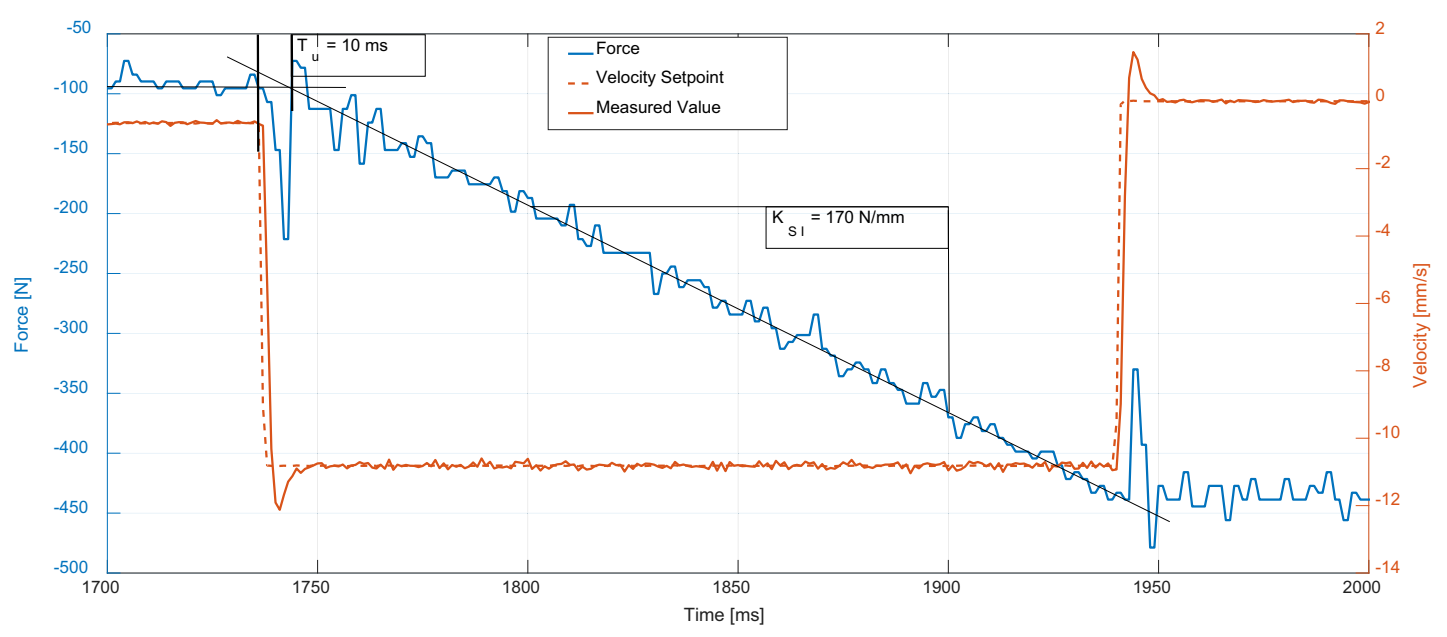

Fig. 5. Identification of the controlled system. 


\subsection{Adjustment instructions}

There is a variety of methods and procedures available for adjusting the controller. However, corresponding specifications and boundary conditions must be taken into account. An integrating behavior with one delaying time constant was determined in the identification of the controlled system. Compliant with the calculation according to the symmetrical optimum, PI-controllers or PID-controllers are suitable for this purpose [12]. The adjustment instruction according to the symmetrical optimum is advantageous in the case of disturbances and is often used, in particular, for force control loops. If the parameters of the controlled system are known, the method can be used without much mathematical effort. On the basis of the identified system parameters, the controller parameters could be calculated. These are summarized together with the formulas in Table 3.

Table 3. Calculated controller parameter.

\begin{tabular}{|c|c|c|}
\hline $\begin{array}{c}\text { Controller } \\
\text { type }\end{array}$ & Setting rule & $\begin{array}{c}\text { Controller } \\
\text { parameter }\end{array}$ \\
\hline \multirow{3}{*}{ PI } & $\mathrm{T}_{\mathrm{N}}=\mathrm{a}^{2 *} \mathrm{~T}_{\mathrm{u}}$ & $\begin{array}{c}\mathrm{K}_{\mathrm{I}}=\mathrm{K}_{\mathrm{P}} / \mathrm{T}_{\mathrm{N}}= \\
4.819\left[\mathrm{~mm} /\left(\mathrm{N}^{*} \mathrm{~s}^{2}\right)\right]\end{array}$ \\
\cline { 2 - 3 } & $\mathrm{K}_{\mathrm{P}}=1 /\left(\mathrm{a} * \mathrm{~K}_{\mathrm{SI}} * \mathrm{~T}_{\mathrm{u}}\right)$ & $\begin{array}{c}\mathrm{K}_{\mathrm{P}}= \\
1.205[\mathrm{~mm} /(\mathrm{N} * \mathrm{~s})]\end{array}$ \\
\hline
\end{tabular}

First, a PI-controller was selected for the experiments. The factor $a$ is a calculation coefficient, in which the damping of the system is included. It is calculated to:

$$
a=2 * D+1
$$

The damping $\mathrm{D}$ was determined as 2 . This shall allow a fast balancing with moderate overshoot.

\section{Experimental and evaluation}

The force control can be realized on the mechanically coupled drives with various embodiments. On the one hand, it is possible to control both drives separately to a force setpoint. However, positional differences occur here with high probability, which leads to a tilt of the plunger. This variant is therefore only suitable for applications in which tilting is permitted or where the differences are very small respectively tolerable. The demonstrator is suitable for such scenarios.

In the second variant, the master axis is in the forcecontrolled state, while the slave axis is controlled to the actual position value of the master axis. As a result, the parallelism of the plunger can be ensured. This is the much more relevant use case. Therefore, the investigation is first focused on this variant. The comparison and evaluation of the parameterization for the controller is based on quality criteria (control rise time, overshoot, settling time) in the time domain. For this purpose, a force jump is switched to the closed loop and the step response is detected. Thus, there is first a direct activation respectively a changeover of the force control. For this reason, the spring is preloaded. Subsequently, the jump with a height of $300 \mathrm{~N}$ is switched on manually.

In this way, the determined parameters for the force controller, summarized in Table 3, were examined on the demonstrator. It turned out that this parameter setting is not suitable for force control. There were strong vibrations and the control loop was unstable. For this reason, an empirical adjustment of the force control parameters was carried out. For this purpose, a Pcontroller and two PI-controllers, which were supplemented by an actual value filter with a time constant $T_{\mathrm{f}}=10 \mathrm{~ms}$, were analyzed. Their characteristic values are summarized in Table 4.

Table 4. Controller parameter.

\begin{tabular}{|c|c|}
\hline $\begin{array}{c}\text { Controller } \\
\text { type }\end{array}$ & Controller parameter \\
\hline \multirow{2}{*}{$\mathrm{P}$} & $\mathrm{K}_{\mathrm{P}}=7.28 \mathrm{e}-002\left[\mathrm{~mm} /\left(\mathrm{N}^{*} \mathrm{~s}\right)\right]$ \\
\hline \multirow{2}{*}{$\mathrm{PI}(1)$} & $\mathrm{K}_{\mathrm{P}}=5.6 \mathrm{e}-002\left[\mathrm{~mm} /\left(\mathrm{N}^{*} \mathrm{~s}\right)\right]$ \\
\cline { 2 - 2 } & $\mathrm{K}_{\mathrm{I}}=3.4 \mathrm{e}-002\left[\mathrm{~mm} /\left(\mathrm{N}^{*} \mathrm{~s}^{2}\right)\right]$ \\
\hline \multirow{2}{*}{$\mathrm{PI}(2)$} & $\mathrm{K}_{\mathrm{P}}=7.28 \mathrm{e}-002\left[\mathrm{~mm} /\left(\mathrm{N}^{*} \mathrm{~s}\right)\right]$ \\
\cline { 2 - 2 } & $\mathrm{K}_{\mathrm{I}}=6.8 \mathrm{e}-002\left[\mathrm{~mm} /\left(\mathrm{N}^{*} \mathrm{~s}^{2}\right)\right]$ \\
\hline
\end{tabular}

Figure 6 compares the curves of the three controller

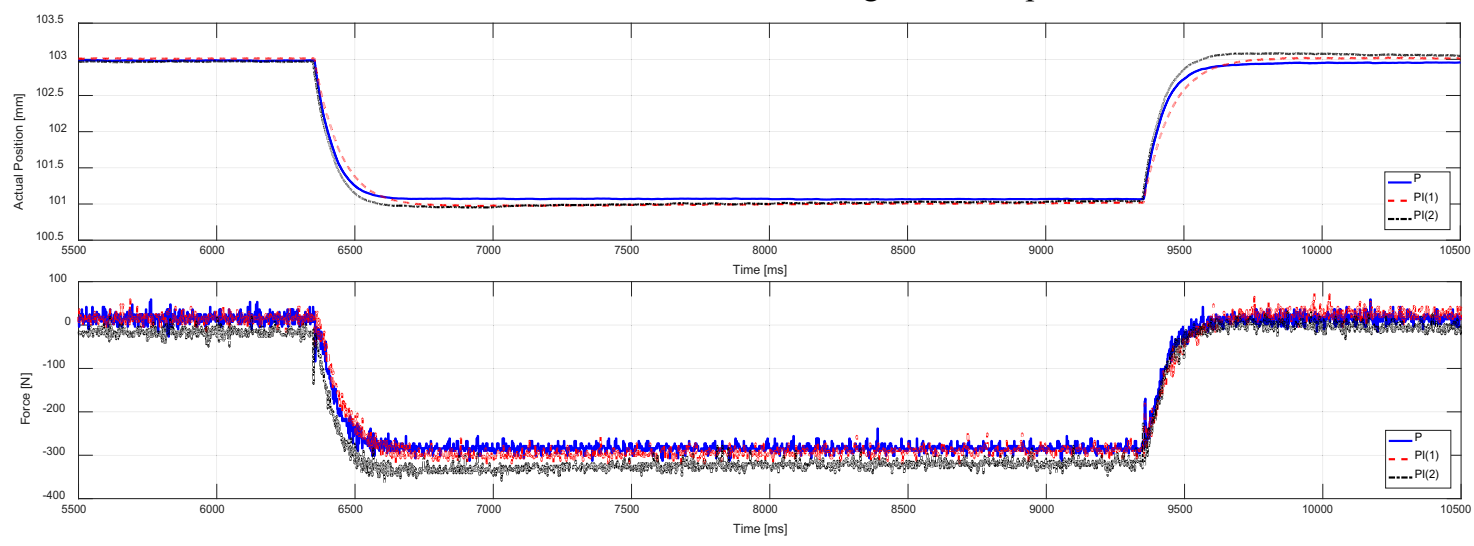

Fig. 6. Comparison of controller performance. 
settings in the time domain for drive 1. Both, the actual position and the force are displayed. A force jump of $300 \mathrm{~N}$ was applied to the system again, in order to assess the controller behavior. The force jump was applied to the preloaded spring assembly. This results in slight deviation in the starting value of the force jump. A difficulty for the investigation and evaluation of the course of forces is the noise. This results from the measuring range and the resolution of the force sensors.

Both PI-controller show a slight overshoot in the actual position as well as in the force value. In addition, the setting $\mathrm{PI}(2)$ is faster due to its higher I-component. However, the I-component in the PI-controller is relatively low, which is why only small differences occur in the overshoot and in the dynamics compared to the P-controller. Therefore, they can also be labeled as a PI-controller with dominant P-component. The exact values for assessing the control performance can be found in Table 5 .

Table 5. Comparison of quality criteria.

\begin{tabular}{|c|c|c|c|}
\hline $\begin{array}{c}\text { Controller } \\
\text { typ }\end{array}$ & $\begin{array}{c}\text { Control rise } \\
\text { time }\end{array}$ & $\begin{array}{c}\text { Settling } \\
\text { time }\end{array}$ & overshoot \\
\hline P & $220[\mathrm{~ms}]$ & $220[\mathrm{~ms}]$ & $0[\mathrm{~N}]$ \\
\hline PI (1) & $220[\mathrm{~ms}]$ & $1320[\mathrm{~ms}]$ & $20[\mathrm{~N}]$ \\
\hline PI (2) & $166[\mathrm{~ms}]$ & $1650[\mathrm{~ms}]$ & $25[\mathrm{~N}]$ \\
\hline
\end{tabular}

On the basis of these results, it is recommended to implement the P-controller for further investigations at the demonstrator with the described control structure.

\section{Summary and future work}

Constantly increasing demands on the productivity and the quality of manufacturing processes always require new research efforts. The control of process forces has considerable potential for improvement in the area of forming technology. At the same time, position control is essential for compliance with defined position specifications in many applications. Thus, the drafting of sophisticated control concepts, which enable joint use or the combination of force and position control, is the focus of future developments.

The approach presented in this paper aims to develop a hybrid control concept for forming operations that is suitable to control process forces and the position simultaneously. Therefor a force control was designed at a servo forming unit with two coupled drives in a first step. It could be shown that a force control, which is superimposed on a speed control loop with subordinate current control loop, can be integrated into the control architecture. In addition various controller types and adjustment rules were experimentally investigated and compared on the basis of corresponding quality criteria. It turned out that an empirical design achieves the best results. Furthermore, a P-controller prevailed against the investigated PI-controllers in terms of performance.
Despite the promising results, there is a clear need for further investigation in the future. Examination fields are especially methods and possibilities for the combination of force and position control as well as the controller design and parameterization. The positioning and integration of the force controller in the cascade structure is of interest, too. In future work, the other variants and their effects will be examined. In this context, novel control algorithms and methods such as prediction, adaptation or cognition should be considered, too. Furthermore, the effects of problematic load situations, such as an eccentric force application, must be investigated. Moreover, an integration of sensors into the tool is necessary for different use cases, in order to detect the current process variable. In this context, suitable concepts for the integration of the measuring signals must be investigated and developed.

\section{Acknowledgement}
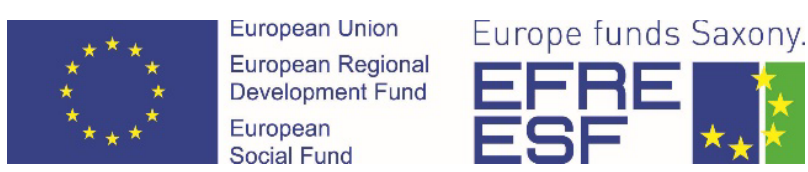

Funded by the European Union (European Social Fund) and the Free State of Saxony.

\section{References}

1. D. Schröder. Elektrische Antriebe - Regelung von Antriebssystemen 2. Auflage. Springer Verlag. Berlin (2001)

2. J. M. Allwood, et al., Closed-loop control of product properties in metal forming. CIRP Annals Manufacturing Technology, 65: 573-596 (2016)

3. A.G. Ulsoy, Y. Koren. Control of Machining Process. Journal of Dynamic Systems Measurement and Control 115: 301-308 (1993)

4. G. Chryssolouris, S. Patel. In Process Control for Quality Assurance. In: D. Tijunelis, K. McKee. Manufacturing High Technology Handbook, Marcel Dekker, New York, 609-643 (1987)

5. R.G. Landers, A.G. Ulsoy, R.J. Furness. Process monitoring and control of machining operations. In: O. Nwokah, Y. Hurmuzlu. The mechanical systems design handbook, CRC Press, Boca Raton, 85-101 (2002)

6. Y. Liu, T. Cheng, L. Zuo. Adaptive control constraint of machining processes. The International Journal of Advanced Manufacturing Technology, 17(10): 720-726 (2001)

7. R.G. Landers, A.G. Ulsoy, Y.-H. Ma. A comparison of model based machining force control approaches. International Journal of Machine Tools and Manufacture 44(7-8): 733-748 (2004)

8. A. Sewohl, M. Rehm, H. Schlegel, M. Putz. Konzepte zur hybriden Kraft-Lageregelung an Produktionsmaschinen. Kolloquium vom Werkstoff 
zum ressourcenschonenden Produkt, Chemnitz (2018)

9. D. Landgrebe, et al. Tooling Flexibility = Flexibility in Forming Technology?!, Proceedings of 5th International Conference on Accuracy in Forming Technology, Chemnitz, November: 10-11, (2015)

10. Exlar Cooperation. GS Series Linear Actuators with Integrated manual,
https://exlar.com/de/produkt/gsx-serie/.Exlar Cooperation (2013)

11. O. Zirn. Machine Tool Analysis - Modelling, Simulation and Control of Machine Tool Manipulators. Habilitation, ETH Zürich (2008)

12. H. Lutz, W. Wendt. Taschenbuch der Regelungstechnik, Wissenschaftlicher Verlag Harri. Frankfurt am Main (2012). 